\title{
SIRT1 negatively regulates amyloid-beta-induced inflammation via the NF-кB pathway
}

\author{
L. Cao ${ }^{1 *}$, C. Liu $^{1 *}$, F. Wang ${ }^{2}$ and H. Wang ${ }^{1}$ \\ ${ }^{1}$ Department of Ophthalmology, Tenth People's Hospital, School of Medicine, Affiliate of Tongji University, Shanghai, China \\ ${ }^{2}$ Department of Ophthalmology, Shanghai Tenth People's Hospital, Shanghai, China
}

\begin{abstract}
Chronic inflammation induced by amyloid-beta $(A \beta)$ plays a key role in the development of age-related macular degeneration (AMD), and matrix metalloproteinase-9 (MMP-9), interleukin (IL)-6, and IL-8 may be associated with chronic inflammation in AMD. Sirtuin 1 (SIRT1) regulates inflammation via inhibition of nuclear factor-kappa B (NF-kB) signaling, and resveratrol has been reported to prevent $A \beta$-induced retinal degeneration; therefore, we investigated whether this action was mediated via activation of SIRT1 signaling. Human adult retinal pigment epithelial (RPE) cells were exposed to A $\beta$, and overactivation and knockdown of SIRT1 were performed to investigate whether SIRT1 is required for abrogating A $\beta$-induced inflammation. We found that A $\beta$-induced RPE barrier disruption and expression of IL-6, IL-8, and MMP-9 were abrogated by the SIRT1 activator SRT1720, whereas alterations induced by A $\beta$ in SIRT1-silenced RPE cells were not attenuated by SRT1720. In addition, SRT1720 inhibited $A \beta$-mediated NF- $\kappa B$ activation and decrease of the NF- $\kappa B$ inhibitor, $I \kappa B \alpha$. Our findings suggest a protective role for SIRT1 signaling in A $\beta$-dependent retinal degeneration and inflammation in AMD.
\end{abstract}

Key words: Amyloid-beta; SRT1720; Age-related macular degeneration; Barrier integrity; Tight junction; Matrix metalloproteinase-9

\section{Introduction}

Age-related macular degeneration (AMD) is a principal cause of irreversible blindness in people over 65 years of age in industrialized countries. AMD is a multifactorial disease and its pathogenesis remains unexplored; however, recent evidence confirms that inflammation plays an important role in its pathology (1). Drusen, tiny yellow or white accumulations of extracellular material within the retina peculiar to AMD, have been suggested as high-risk factors for the development of chronic inflammation. $(1,2)$. Amyloid-beta $(A \beta)$, a known constituent of drusen, is thought to contribute to development of AMD (3-5).

The retina is an immune-privileged site protected by a local blood-retinal barrier (BRB), but it is not known how immune cells can pass through this barrier and cause chronic inflammation. A layer of retinal pigment epithelial (RPE) cells forms the outer BRB, and the inner BRB is formed by blood vessels of the inner retina. Alterations of the BRB play a crucial role in the development of AMD $(6,7)$. The function of the outer BRB is dependent on welldeveloped tight junctions (TJs) between RPE cells, which are mediated by the transmembrane proteins occludin and the claudin family, and junctional adhesion molecules, along with the scaffolding zonula occludens protein-1 (ZO-1). Abnormal expression or localization of occludin and ZO-1 in RPE cells leads to barrier dysfunction $(8,9)$. The antioxidant resveratrol has been shown to protect ARPE-19, a spontaneously transformed RPE cell line, from $A \beta$-induced barrier dysfunction (10). Our previous study found that matrix metalloproteinase- 9 (MMP-9) mediated $A \beta$-induced barrier disruption, suggesting that MMP-9 contributes to chronic inflammation in AMD (11). Breakdown of the epithelial barrier would thus be both a stimulus for inflammation in tissue injury and a component of normal inflammatory processes that permit leukocyte influx into areas of tissue damage.

Colocalization of $A \beta$ and activated complement immunoreactivity in AMD patients provide evidence for $A \beta$ deposition in the local inflammatory events contributing to pathogenesis of AMD (12), and suggest that $A \beta$-induced complement activation may be involved in chronic inflammation in AMD (13). Microarray analysis of gene expression in ARPE-19 revealed that $A \beta$ stimulation

Correspondence: F. Wang, Department of Ophthalmology, Shanghai Tenth People's Hospital, No. 301 Middle Yan Chang Road, Shanghai 200072, China. E-mail: milwang_122@msn.com

${ }^{*}$ These authors contributed equally to this study.

Received February 4, 2013. Accepted May 28, 2013. First published online August 26, 2013. 
significantly upregulated interleukin (IL)-8 gene expression (14). Our previous study found that $A \beta$ stimulation increased IL-6, IL-8, and MMP-9 expression in human adult RPE cells (11). Increased intraocular concentrations of IL-6, IL-8, and MMP-9 have also been found in AMD patients, and the levels were significantly associated with the severity of the disease (15). However, the mechanisms by which $A \beta$ upregulates inflammation-associated cytokines is largely unknown.

Nuclear factor-kappa B (NF-кB), a key regulator of the inflammation response, is modulated by post-translational modifications, including reversible acetylation of the NF-kB RelA/p65 subunit (16). Full transcriptional activity of RelA/ p65 requires acetylation of Lys310, which can be deacetylated by sirtuin 1 (SIRT1) (17). Resveratrol, an activator of SIRT1 (18), inhibits NF-kB signaling by promoting deacetylation of Lys310 of ReIA/p65 (16). Thus, SIRT1 may be a key regulator of inflammation in mammalian cells via inhibition of $\mathrm{NF}-\kappa \mathrm{B}$ activation. In this study, we explored the inhibitory role of SIRT1 in A $\beta$-induced proinflammatory cytokine production and BRB disruption in human adult RPE cells by activating SIRT1 with SRT1720 or silencing SIRT1 with small-interfering RNA (siRNA). We further demonstrated that $A \beta$-induced expressions of MMP-9, IL-8, and IL-6 were regulated by the SIRT1/NF-kB pathway.

\section{Material and Methods}

\section{$A \boldsymbol{\beta}_{1-42}$ oligomerization and detection of $A \boldsymbol{\beta}_{1-42}$ oligomers}

Oligomerized $A \beta(O A \beta)$ was synthesized as previously described (11). Briefly, lyophilized $A \beta_{1-42}$ peptide (SigmaAldrich, USA) was dissolved in $1.5 \mathrm{M}$ hexafluoroisopropanol (HFIP) on ice and aliquoted at $-20^{\circ} \mathrm{C}$. $A \beta$ monomers were spin-vacuumed just prior to use, diluted to $250 \mu \mathrm{M}$ in HFIP solution, and kept at room temperature for 3 days to synthesize $A \beta$ oligomers.

Several lines of evidence have demonstrated that soluble $A \beta$ oligomers may be better correlated with the severity of the disease than are monomers or insoluble amyloid fibrils $(3,19)$. Therefore, the oligomeric form of $A \beta_{1-42}$ was verified using Western blot with the anti-A $\beta$ monoclonal antibody 6E10 (1:500, Covance, USA).

\section{Ethics}

Informed consent for human tissue donation was obtained from relatives, and the study protocol was approved by the Ethics Committee of the Tenth People's Hospital affiliated to the Tongji University and adhered to the tenets of the Declaration of Helsinki for experiments involving human tissue.

\section{Isolation of human RPE cells}

Five human donor eyes were obtained from the eye bank of the Eye \& ENT Hospital of Fudan University, Shanghai, China. The donors were between 30 and 40 years of age and none had a history of eye disease. Human RPE cells were harvested as described previously (11). In brief, whole eyes were cleaned in $0.9 \% \mathrm{NaCl}$ solution, immersed in $5 \%$ polyvinylpyrrolidone iodine, and rinsed again in $\mathrm{NaCl}$ solution. The anterior segment was removed from each eye and the neural retina was peeled away from the RPE choroid sclera. The eyecup was rinsed with $\mathrm{Ca}^{2+}$ and $\mathrm{Mg}^{2+}$-free Hank's balanced salt solution, and treated with $0.25 \%$ trypsin for $1 \mathrm{~h}$ at $37^{\circ} \mathrm{C}$. The trypsin was aspirated and replaced with DMEM/F12 (HyClone Laboratories, USA) supplemented with $20 \%$ fetal calf serum. The RPE cells isolated from the five donor eyes were combined, yielding about $10^{5}$ cells per milliliter.

\section{Silencing of SIRT1 by RNA interference}

To confirm the exact role of SIRT1 on MMP-9, IL-8, and IL- 6 expression by OA $\beta$-stimulated cells, gene expression of SIRT1 was silenced by an RNA interference strategy. The SIRT1 silencer and negative siRNA were synthesized by Genepharma (China). The sequences were: SIRT1 siRNA, sense: 5'-GAUGAAGUUGACCUCC UCATT-3', anti-sense: 5'-UGAGGAGGUCAACUUC AUCTT-3'; and negative siRNA (siRNA-N), sense: 5'-CCUACGCCACCAAUUUCGU-3', anti-sense: 5'-ACG AAAUUGGUGGCGGUAGG-3'. siRNA at a final concentration of $25 \mathrm{nM}$ was combined with $10 \mu \mathrm{L}$ Lipofectamine 2000 (Invitrogen, USA) in $500 \mu \mathrm{L}$ DMEM/F12 and allowed to complex by incubation for $20 \mathrm{~min}$. The RPE cells were incubated with the transfection mixture for $24 \mathrm{~h}$, and the cells were transferred to culture medium as described above and incubated for $72 \mathrm{~h}$ before treatment.

\section{Cell viability assay}

Human adult RPE cells $\left(2 \times 10^{4} /\right.$ well $)$ seeded on 96well plates were treated with $0.001-12.0 \mu \mathrm{M}$ OA $\beta$ for $24 \mathrm{~h}$ or treated with $0.3 \mu \mathrm{M} \mathrm{OA} \beta$ for 4-24 $\mathrm{h}$. To determine its protective effect, cells were treated with $5 \mu \mathrm{M}$ SRT1720 (Cayman Chemical, USA) $1 \mathrm{~h}$ before or $1 \mathrm{~h}$ after treatment with $0.3 \mu \mathrm{M} O A \beta$. In other experiments, RPE cells exposed to the transfection mixture $(25 \mathrm{nM}, 24 \mathrm{~h})$ were treated with OA $\beta$ with or without SRT1720. Cell viability was measured by addition of 3-(4,5-dimethylthiazol2-yl)-5-(3-carboxymethoxyphenol)-2-(4-sulfophenyl)-2Htetrazolium (MTS, Promega, USA) for $3 \mathrm{~h}$ at $37^{\circ} \mathrm{C}$. The absorbance was measured spectrophotometrically at $490 \mathrm{~nm}$ with a microplate reader (ELx800, Bio-Tek, USA).

\section{Real-time quantitative-PCR}

Total RNA was isolated using Trizol (Invitrogen) and reverse-transcribed to cDNA with RT Master Mix (TaKaRa, China). Expression of IL-8 and IL-6 was measured using a PCR mix (TaKaRa). The PCR primers were: IL-8, sense: 5'-GACATACTCCAAACCTTTCCAC-3', antisense: 5'-AAACTTCTCCACAACCCTCTG-3'; IL-6, sense: 5'-CACTCACCTCTTCAGAACGAAT-3', antisense: 5'-TTTGTACTCATCTGCACAGCTC-3'. An ABI7500 
RT-PCR system (Applied Biosystems, USA) was used. The RT-PCR results were normalized against $\beta$-actin (sense: $5^{\prime}$ TGGGCATGGGTCAGAAGGATTCC-3', antisense: 5' CCACACGCAGCTCATTGTAGAAGG-3'), which served as the control, and relative gene expression was reported as "fold-change", calculated using the $\Delta \Delta \mathrm{Ct}$ method.

\section{Gelatin zymography}

Gelatin zymography was carried out as previously described (11). Briefly, supernatant was collected after treatment and subjected to $10 \%$ SDS-PAGE with $1 \mathrm{mg} / \mathrm{mL}$ gelatin. After electrophoresis, gels were incubated in $2.5 \%$ Triton $\mathrm{X}-100\left(1 \mathrm{~h}, 37^{\circ} \mathrm{C}\right)$ followed by overnight incubation in $50 \mathrm{mM}$ Tris- $\mathrm{HCl}$, pH 7.8, $5 \mathrm{mM} \mathrm{CaCl}_{2}, 0.02 \% \mathrm{NaN}_{3}$, and $0.02 \%$ Brij. Gels were stained with $2.5 \%$ Coomassie blue R-250 (Bio-Rad, USA) for 45 min followed by destaining in deionized water with $10 \%$ acetic acid and $20 \%$ methanol. Gels were scanned and band densities were measured using Photoshop CS4.0 (Adobe Systems, USA).

\section{Cell morphology and immunofluorescence staining}

To confirm the protective role of SIRT1 on RPE barrier integrity, normal RPE cells or siRNA-transfected RPE cells were treated with $0.3 \mu \mathrm{M} \mathrm{A} \beta$ for $24 \mathrm{~h}$, with or without pretreatment with $5 \mu \mathrm{M}$ SRT1720 for $1 \mathrm{~h}$. After 3 days, the cells were fixed in $4 \%$ paraformaldehyde for 30 min and blocked with $1 \%$ bovine serum albumin in Trisbuffered saline for $1 \mathrm{~h}$, then incubated with rabbit antioccludin antibody (1:250), mouse anti-ZO-1 antibody (1:250; Abcam, China), or rabbit anti-ezrin antibody (1:250; Anbo Biotechnology, USA) for 1 h. After washing, they were incubated with AlexaFluor 488-conjugated or AlexaFluor 594-conjugated secondary antibody (1:500; Invitrogen) and DAPI nuclear stain (1:1000) for $1 \mathrm{~h}$. Slides were then viewed on a Leica TCS SP5 scanning confocal microscope (Leica Microsystems, Germany). For immunofluorescence analysis of nuclear translocation of NF$\kappa \mathrm{B} / \mathrm{p} 65$, the cells were incubated with rabbit polyclonal anti-p65 primary antibody (1:500, Abcam) for $1 \mathrm{~h}$ and incubated with anti-rabbit AlexaFluor 488-conjugated secondary antibody and DAPI (1:1000) for $1 \mathrm{~h}$.

\section{Measurement of transepithelial electrical resistance (TER)}

Normal or siRNA-transfected RPE cells were grown on a microporous filter to form monolayers. TER was measured using a Millicell electrical resistance system (Millipore, USA) and calculated by subtracting the value of a blank filter without cells from the experimental value. Final resistance $\times$ area products $\left(\Omega \times \mathrm{cm}^{2}\right)$ were obtained by multiplying TER by the effective growth area. Fifteen days after TER stabilization, RPE monolayers were exposed to $0.3 \mu \mathrm{M} O A \beta$ for $24 \mathrm{~h}$ in the presence or absence of $5 \mu \mathrm{M}$ SRT1720 for $1 \mathrm{~h}$. The culture medium was then changed, restoring normal conditions, and TER was measured after 3 days of incubation. Measurements were repeated at least four times for each filter, and each experiment was repeated at least four times.

\section{Permeability assay}

Permeability assays were performed by measuring passive permeation of fluorescein isothiocyanate (FITC)dextran (4 kDa; Sigma-Aldrich) across confluent cells grown on filters. Fifteen days later, the monolayers were treated as described above, and $500 \mathrm{mg} / \mathrm{mL}$ FITCdextran was added to the upper chamber after treatment. Samples $(100 \mu \mathrm{L})$ were taken from the upper and lower chambers $24 \mathrm{~h}$ after addition of FITC-dextran. The concentration of FITC-dextran in the samples was measured with a microplate reader (ex1800; Biotek, USA). Each experiment was repeated four times.

\section{Protein extraction and Western blot analysis}

To explore the protective mechanism of SRT1720, protein levels of NF-kB, nuclear factor of kappa light polypeptide gene enhancer in B-cells inhibitor, alpha

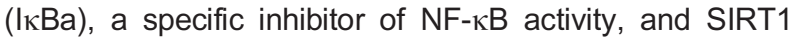
were analyzed by Western blot. Briefly, RPE cells were pretreated with $5 \mu \mathrm{M}$ SRT1720 for $60 \mathrm{~min}$, then the medium was changed immediately with $0.3 \mu \mathrm{M} \mathrm{A} \beta$ for $4 \mathrm{~h}$. Total proteins were obtained using radio-immunoprecipitation assay buffer (Abcam) containing proteinase inhibitor, and nuclear proteins were extracted following the manufacturer's protocol. Protein content was determined by a bicinchoninic acid assay (Pierce, USA). Equal amounts of total cellular protein were loaded per lane onto $10 \%$ SDSPAGE for electrophoresis and then transferred onto PVDF membranes. The membranes were blocked, and incubated with the primary antibody, rabbit polyclonal antibodies

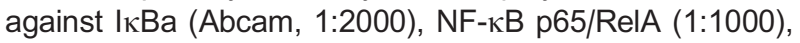
or SIRT1/Sir2 (1:2000), and rabbit polyclonal antibodies against glyceraldehyde-3-phosphate dehydrogenase (1:2000) or TATA binding protein (1:1000), overnight. The membranes were then washed and incubated with horseradish peroxidase-coupled secondary antibodies for $2 \mathrm{~h}$. Blots were washed and developed with chemiluminescent reagent. Membranes were exposed to ImageQuant LAS 4000, and densitometry was performed using Photoshop CS4.0.

\section{Statistical analysis}

Results are reported as means $\pm S E$. The unpaired $t$-test or ANOVA was used for statistical analysis (SPSS 15.0, USA) and differences were considered to be significant at $\mathrm{P}<0.01$ or $\mathrm{P}<0.05$.

\section{Results}

\section{Characterization of $A \beta_{1-42}$ oligomers and morphology} of human adult RPE cells

$A \beta$ samples were examined after incubation for 12 or $72 \mathrm{~h}$. Spherical oligomers, including trimers and 
tetramers, were initially absent from freshly prepared $A \beta_{1 \text { - }}$ 42 solutions or monomers (aggregated for less than $24 \mathrm{~h}$ ). Western blots with anti-A $\beta$ antibody (6E10) further confirmed the presence of $A \beta_{1-42}$ as a monomer, trimer, and tetramer in the oligomerized $A \beta$ sample, whereas only monomers were detected in the fresh non-oligomerized $A \beta$ sample (Figure $1 A$ ). These results indicated that the $A \beta$ samples incubated for $72 \mathrm{~h}$ had aggregated, forming oligomers.

Structure integrity is the basis of good function. To confirm that the isolated human adult RPE displayed classic morphology (uniform hexagonal arrays of cells), confluence, and uniform pigmentation typical of native tissue, the morphology of the cells was evaluated by fluorescence microscopy. Our previous data confirmed that isolated human adult RPE cells were heavily pigmented and had the hexagonal epithelial morphology seen in native tissue (11). Ezrin is a cytoskeleton protein that has been identified exclusively in RPE cell apical processes and has been associated with polarization of RPE cells (20). Apical localization of ezrin in cultured human adult RPE cultures was confirmed by fluorescence microscopy (Figure 1B).

\section{SIRT1 was required to protect RPE cells from A $\beta$ - induced cytotoxicity}

The effects of $0.001-12.0 \mu \mathrm{M}$ OA $\beta$ on cell viability were observed at $24 \mathrm{~h}$ (Figure $2 \mathrm{~A}$ ); mean cell viability was $95 \%$ at $0.1 \mu \mathrm{M}$ but fell to $30 \%$ at $12.0 \mu \mathrm{M}$. The effect of $0.3 \mu \mathrm{M}$ OA $\beta$ on the viability of cells was assayed at 4-24 h; cell survival gradually dropped from $87.6 \%$ at $4 \mathrm{~h}$ to $68.4 \%$ at $24 \mathrm{~h}$ (Figure 2B). This indicated that a timeand dose-dependent decrease in cell viability occurred in OA $\beta$-stimulated cells. Treatment with $5 \mu \mathrm{M}$ SRT1720 $1 \mathrm{~h}$ before or $1 \mathrm{~h}$ after $0.3 \mu \mathrm{M}$ OA $\beta$ treatment for $24 \mathrm{~h}$ increased cell viability by $33 \%(\mathrm{P}<0.05)$ or $26 \%$ $(P<0.01)$, respectively, compared with the OA $\beta$-treated group $(\mathrm{P}<0.01)$. However, inhibition of SIRT1 with SIRT1specific siRNA resulted in an increased susceptibility of RPE cells to OA $\beta$ stimulation and significantly attenuated SRT1720's protection against OA $\beta$ (Figure 2C). This suggested that SRT1720 might protect RPE cells from OA $\beta$-induced cytotoxicity via the SIRT1 pathway. To confirm this result, we examined SIRT1 expression in
Western blots. As shown in Figure 2D, SIRT1 expression was significantly decreased 2 -fold in the OA $\beta$ insult group compared with controls $(\mathrm{P}<0.05)$. Meanwhile, the expression of SIRT1 was markedly increased when RPE cells were incubated with SRT1720 ( $<<0.01$; Figure 2D). In addition, knockdown of SIRT1 completely blocked the effect of SRT1720 on SIRT1, and transfection of cells with siRNA-N did not abolish the positive effect of SRT1720 on SIRT1 activation (Figure 2D). These data suggested that SIRT1 activation was essential for protection of RPE cells against $O A \beta$ injury.

\section{SIRT1 negatively regulates OA $\beta$-induced inflammation and MMP-9 expression}

We have previously shown that $O A \beta$ could increase IL-8, IL-6, and MMP-9 expression in human adult RPE cells (11). SRT1720, a potent SIRT1 agonist, has been reported to have anti-inflammatory properties (21). Therefore, we used SRT1720 to investigate the antiinflammation function of SIRT1. For inhibition studies, SIRT1 expression was silenced by RNA interference. To demonstrate the anti-inflammation effects of SIRT1, we measured the expression levels of IL-8, IL-6, and MMP-9 using RT-PCR and gelatin zymography. Treatment with SRT1720 significantly attenuated OA $\beta$-induced upregulation of IL-6, IL-8, and MMP-9, whereas the inhibitory effects of SRT1720 on OA $\beta$-induced upregulation of IL-6, IL-8, and MMP-9 were attenuated in the cells in which SIRT1 expression was knocked down (Figure 3). To test the time course of inflammation inhibition, SRT1720 was administered $1 \mathrm{~h}$ before or $1 \mathrm{~h}$ after $O A \beta$ treatment. Inflammation was not significantly inhibited following SRT1720 administration $1 \mathrm{~h}$ after OA $\beta$ stimulation compared with SRT1720 pretreatment (Figure 3). These results suggest that SIRT1 is required for blocking the OA $\beta$-induced proinflammatory effect.

\section{SIRT1 is required to attenuate OA $\beta$-induced disruption of barrier integrity}

Structures of occludin and ZO-1 are crucial to the development and maintenance of RPE morphology and function. Ezrin, one of the first proteins to be polarized, is localized mainly on the apical side of well-polarized human RPE cells (20). A recent study found that the

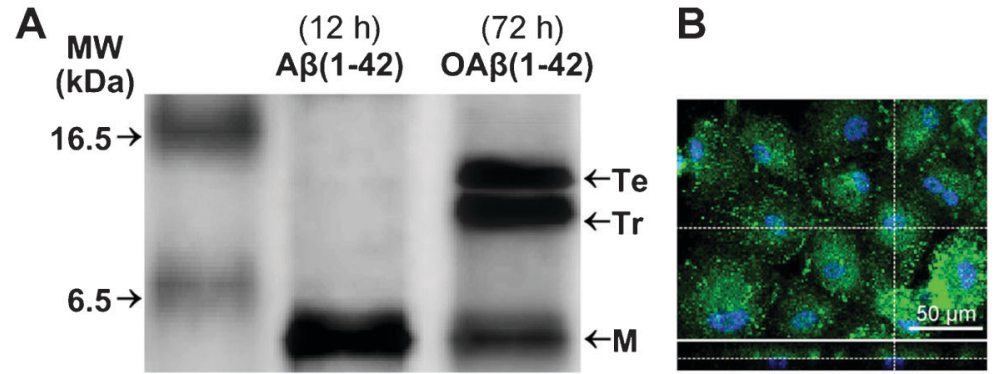

Figure 1. Confirmation of the oligomeric form of $A \beta_{1-42}$ and localization of ezrin in human adult RPE cells. $A$, Oligomerization of $A \beta(1-42)$ was verified by Western blotting. M: monomer of $A \beta_{1-42} ; \mathrm{Tr}$ : trimer of $A \beta_{1-42} ;$ Te: tetramer of $A \beta_{1-42} ; \mathrm{MW}$ : molecular weight. $B$, Immunofluorescence staining for ezrin (green) in human adult RPE cells. The presence of ezrin on the apical side of the RPE microvilli is shown in confocal cross-sectional images (bottom). Nuclei are stained with DAPI (blue). 
A

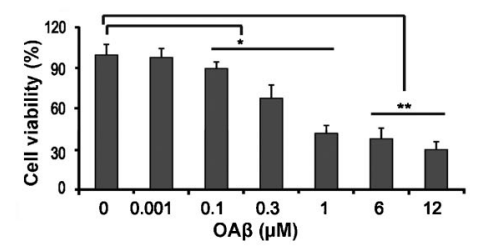

C
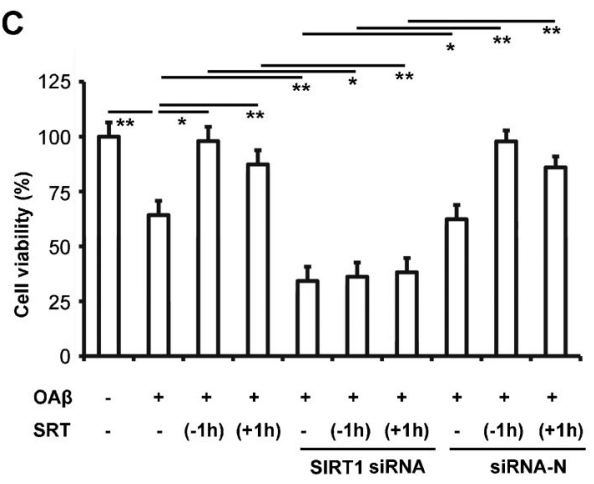

B
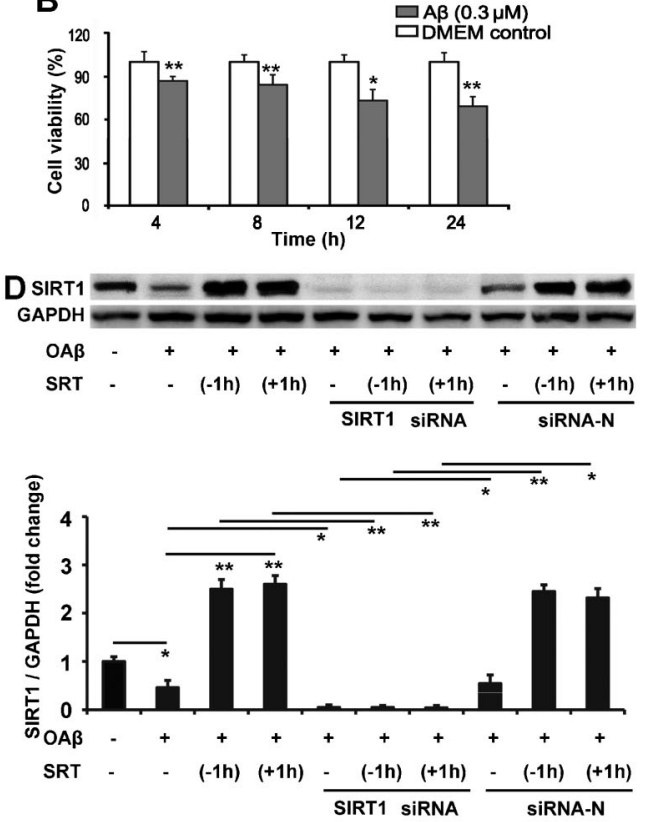

Figure 2. MTS assay of cell viability. A, MTS assay of cell viability after stimulation with different concentrations of OA $\beta$. Upon exposure to $0.001 \mu \mathrm{M} O A \beta$, no reduction of MTS activity was detected in cells, while a significant reduction was observed in response to 0.1 $12 \mu \mathrm{M}$ OA $\beta$ treatment after $24 \mathrm{~h}$. B, MTS assay for cell viability after stimulation with $0.3 \mu \mathrm{M}$ OA $\beta$ for different times. RPE cells stimulated with $0.3 \mu \mathrm{M} \mathrm{OA} \beta$ demonstrated a time-dependent reduction of MTS activity dropping from $87.6 \%$ at $4 \mathrm{~h}$ to $68.4 \%$ at $24 \mathrm{~h}$. $C$, MTS assay on normal or siRNA-transfected cells treated with OA $\beta$ or SRT1720. SRT1720 attenuated OA $\beta$-induced decrease of cell viability, and knockdown of SIRT1 inhibited the protective effect of SIRT1 on cell viability. $D$, Western blot analysis of SIRT1 expression. The SIRT1 expression of OA $\beta$-stimulated cells was lower than that of control, and SRT1720 treatment significantly increased the SIRT1 expression in OA $\beta$-stimulated cells. Transfection with SIRT1 siRNA blocked SIRT1 expression in cells, but transfection with negative siRNA did not affect the inhibitory effect of SRT1720 on OA $\beta$-induced decrease of SIRT1 expression. Data are reported as means \pm SE from 4 measurements. Each panel shows a representative experiment repeated three times with similar results. SRT: cells treated with SRT1720; SIRT1 siRNA: cells transfected with SIRT1 siRNA; siRNA-N: cells transfected with negative control of siRNA. ${ }^{*} \mathrm{P}<0.05$, ${ }^{*} \mathrm{P}<0.01$ (unpaired $t$-test and ANOVA).

SIRT1 activator resveratrol could attenuate OA $\beta$-induced RPE barrier disruption (10). MMP-9 has been reported to modify barrier function by disrupting TJ proteins (22-24), and our previous study demonstrated that silence of MMP-9 partially abrogated the damaging effect of OA $\beta$ on the RPE barrier (11). It has been demonstrated that SIRT1 is a negative regulator of MMP-9 expression in human monocytic cells in vitro (25). Based on the aforementioned result that demonstrated that SIRT1 activation could inhibit OA $\beta$-induced expression of MMP9 (Figure 3), we asked whether SIRT1 could protect RPE cells from OA $\beta$-induced RPE barrier disruption. Normal or siRNA-transfected RPE cells were exposed to OA $\beta$ with or without SRT1720 pretreatment $(1 \mathrm{~h})$. In the control cells (Figure 4A), no morphological change was observed, the distribution of occludin and ZO-1 was continuous and regular with no breaks around the cell borders, and ezrin was localized mainly on the apical side. Exposure to $A \beta$ (Figure 4B) caused a diffuse cytoplasmic distribution of ZO-1 and occludin, and the immune staining of ezrin was dispersed on both sides of the cells. Pretreatment with
SRT1720 (Figure 4C) inhibited the deleterious effects of $A \beta$ on RPE integrity. Knockdown of SIRT1 significantly abolished the protective effect of SRT1720 on OA $\beta$ induced barrier disruption (Figure 4D) compared with siRNA-N-transfected cells. These results suggested that SRT1720, with its anti-inflammatory properties might reverse the deleterious effects of $A \beta$ on RPE barrier structure. However, activation or inhibition of SIRT1 expression could affect the localization of ezrin, and this result implied that ezrin expression or localization was not regulated by SIRT1.

Barrier functional studies, including TER detection and permeability assays, were performed to confirm the protective effect of SIRT1 against the deleterious effect of OA $\beta$ on the RPE barrier. TER (Figure $5 A$ ) was recorded to determine the stability of TJ proteins, and the transepithelial diffusion rate of FITC-dextran (Figure 5B) was measured to evaluate the permeability of the monolayers. A mean TER of $160 \pm 23 \Omega \times \mathrm{cm}^{2}$ was recorded in control cells. OA $\beta$ decreased TER by $83 \pm 18 \Omega \times \mathrm{cm}^{2}$, but pretreatment with SRT1720 partially 


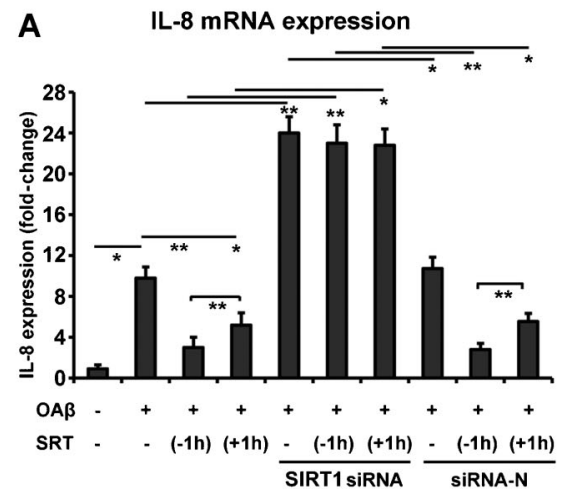

C

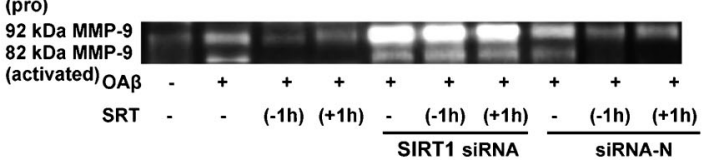

B
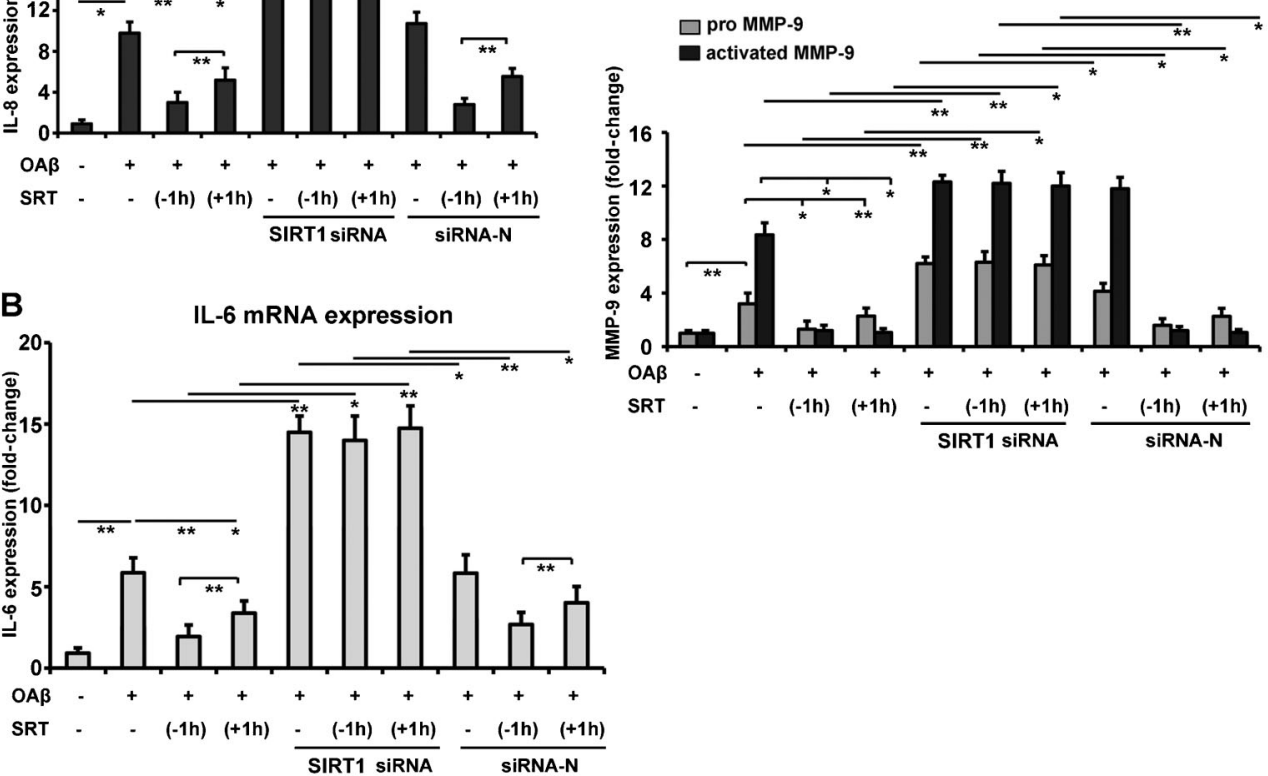

Figure 3. Effects of $A \beta$, SRT1720 and knockdown of SIRT1 on IL-8, IL-6 and MMP-9 expression in RPE cells. A, PCR analysis of IL-8 mRNA expression. $B$, PCR analysis of IL-6 mRNA expression. $C$, Gelatin zymography assay of MMP-9 expression. Stimulation with $0.3 \mu \mathrm{M} \mathrm{OA} \beta$ for $24 \mathrm{~h}$ induced significant increases in mRNA expression of IL-6, IL-8 and MMP-9 in RPE cells. Pretreatment with SRT1720 markedly attenuated OA $\beta$-induced increases of IL-6, IL-8 and MMP-9 expression. Knockdown of SIRT1 gene expression by SIRT1 siRNA significantly intensified the proinflammatory effects of $O A \beta$. Data are from three representative experiments. ${ }^{*} \mathrm{P}<0.05$, ${ }^{* *} \mathrm{P}<0.01$ (unpaired $t$-test and ANOVA).

reversed this effect, and transfection with SIRT1 siRNA significantly exacerbated the $A \beta$-induced decrease of TER (Figure 5A). A significantly increased diffusion rate of FITC-dextran was observed when the cells were stimulated with $A \beta$, but pretreatment with SRT1720 abolished this effect, and SIRT1 knockdown induced a greater permeability than $A \beta$ stimulation alone (Figure $5 B$ ). These results suggested that activation of SIRT1 could prevent $A \beta$-induced barrier dysfunction, including decrease of TER and permeability dysfunction.

\section{Expression of I $\mathrm{kBa}$ and NF-kB in RPE cells}

In the present study, SRT1720 had an obvious effect, protecting RPE cells from $A \beta$-induced cytotoxicity and inflammation and dysfunction of barrier integrity in RPE cells (Figures 2-5). To explore the underlying mechanisms of its beneficial effects, RPE cells were pretreated with SRT1720 for 60 min before OA $\beta$ was added. Expression

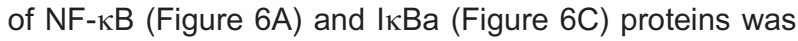
analyzed by Western blot. Nuclear translocation of $\mathrm{NF}-\mathrm{kB} / \mathrm{p} 65$, an index of NF-kB activation, was measured using immunofluorescence (Figure 6B). Treatment of RPE cells with $A \beta$ induced a significant 12.8-fold increase in the amount of NF-kB/p65 in the nucleus (Figure $6 \mathrm{~A}$ ), positive immunostaining of p65 translocated into the nucleus (Figure 6B), and a significant decrease in $1 \kappa \mathrm{Ba}$ protein content (Figure 6C). Pretreatment with SRT1720 decreased the amount of $\mathrm{NF}-\kappa \mathrm{B} / \mathrm{p} 65$ in the nucleus (Figure $6 \mathrm{~A}$ and $\mathrm{B}$ ) and increased the amount of $1 \kappa \mathrm{Ba}$ compared with stimulation with $A \beta$ (Figure $6 \mathrm{C}$ ). Knockdown of SIRT1 with siRNA not only attenuated the inhibitory effect of SRT1720 on A $\beta$-induced nuclear translocation but also induced a higher amount of $N F-\kappa B / p 65$ in the nucleus than the $A \beta$ insult group (Figure 6A and B). With the altered expression levels of SIRT1 (Figure 2D), these results are consistent with the theory that SIRT1 activation has an anti-inflammatory effect via inhibition of NF- $\kappa B$ activation. In addition, we found that the expression level of $I \kappa B \alpha$, an inhibitor of $\mathrm{NF}-\kappa \mathrm{B}$, was increased in SIRT1-siRNA-transfected cells, which implied that the inhibitory effect of SIRT1 on NF-KB was not fully dependent on $I \kappa B \alpha$ activation. 


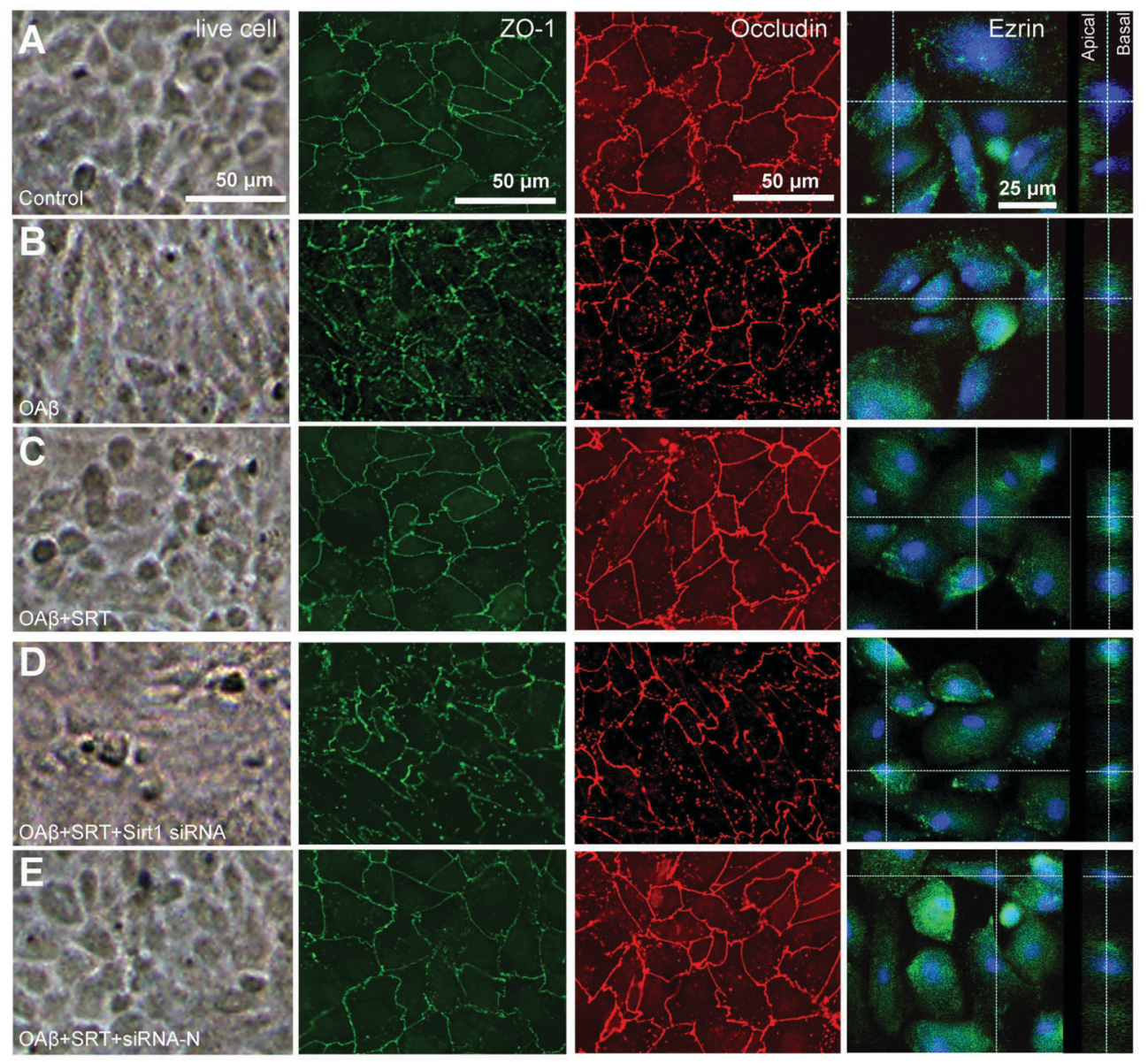

Figure 4. Effects of $A \beta$, SRT1720 and knockdown of SIRT1 on morphology and locations of occludin, ZO-1 and ezrin staining of RPE cells. RPE cells were incubated with varying stimuli for $48 \mathrm{~h}$ as follows: $A$, DMEM/F12 medium; $B, 0.3 \mu \mathrm{M}$ OA $\beta ; C, 5 \mu \mathrm{M}$ SRT1720 for $1 \mathrm{~h}$ before $A \beta$ was added; $D$, SIRT1-silenced RPE cells were incubated with SRT1720 $(5 \mu \mathrm{M}) 1 \mathrm{~h}$ before A $\beta$ was added; $E$, Negative siRNA-transfected RPE cells were incubated with SRT1720 $(5 \mu \mathrm{M}) 1 \mathrm{~h}$ before A $\beta$ was added. Light microscopy showed that cells treated with $\mathrm{OA} \beta(B)$ or transfected with SIRT1 siRNA $(D)$ displayed irregular morphology compared with control $(A)$, but pretreatment with SRT1720 $(C, E)$ reversed the effects induced by $A \beta$. Immunostaining of occludin and ZO-1 showed that the localization of occludin and ZO-1 perfectly matched the typical cobblestone-like morphology of RPE in the control $(A)$ or SRT1720-pretreated cells $(C)$, whereas it showed not only disorganization of the occludin and ZO-1, but also disappearance of typical cobblestone-like morphology in OA $\beta$-treated $(B)$ or SIRT1-silenced cells $(D)$. ZO-1: green; occludin: red; ezrin: green; DAPI: blue; apical: apical side of RPE monolayer; basal: basal side of RPE monolayer.

\section{Discussion}

$\mathrm{OA} \beta_{1-42}$ can induce inflammation and barrier disruption in RPE cells (11), but the underling mechanism is largely unknown. The main results of this study are as follows: 1 ) SIRT1 is required to protect RPE cells from $A \beta$-induced cytotoxicity; 2) SIRT1 negatively regulates $O A \beta$-induced inflammation and MMP-9 expression; 3) SIRT1 is required to attenuate $O A \beta$-induced disruption of barrier integrity; 4) the protective effects of SIRT1 on inflammation and barrier disruption depend on its inhibition of NF-kB.

The present study showed that exposure of human adult RPE cells to $A \beta$ induced a dose- and time-dependent decrease in cell viability (Figure $2 \mathrm{~A}$ and $\mathrm{B}$ ). The protective role of SIRT1 on cell viability was further confirmed by pharmacological activation of SIRT1 with SRT1720 and by the knockdown of SIRT1 gene expression with an RNA interference strategy (Figure 2C). Interestingly, we found that $A \beta$ could inhibit SIRT1 expression (Figure 2D). Accumulated evidence suggests that resveratrol, an SIRT1 activator, can attenuate $A \beta$-induced toxicity (26). $A \beta$-induced MTS reduction as the result of decreased mitochondrial membrane potential is a marker of the production of excessive reactive oxygen species (ROS) 


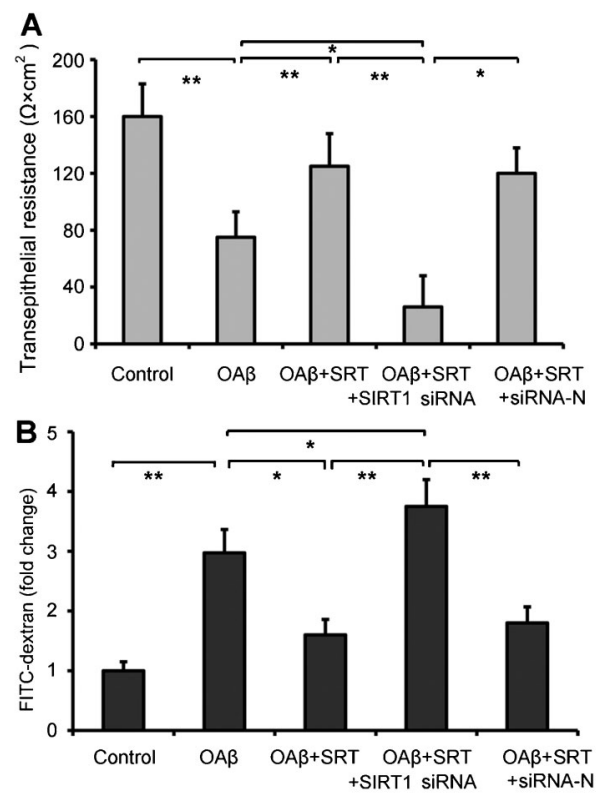

Figure 5. Effects of A $\beta$, SRT1720 and knockdown of SIRT1 on the barrier integrity of RPE monolayers. $A$, Measurement of transepithelial resistance (TER) of retinal pigment epithelial (RPE) cells. $B$, Analysis of transepithelial permeability by measuring the passive permeation of FITC-dextran. Normal or siRNA-transfected RPE cells were cultured for 15 days after TER stabilization, and then left untreated (control) or exposed to $0.3 \mu \mathrm{M}$ OA $\beta$ for $24 \mathrm{~h}$ with or without $5 \mu \mathrm{M}$ SRT1720 pretreatment for $1 \mathrm{~h}$. OA $\beta$ induced a significant decrease in TER and increased permeability compared with the control. SRT1720 markedly attenuated OA $\beta$ induced low TER and high permeability, whereas transfection with SIRT1 siRNA led to a lower TER and a higher permeability than the $\mathrm{OA} \beta$ insult group. Data are from 3 representative experiments. ${ }^{*} \mathrm{P}<0.05,{ }^{* *} \mathrm{P}<0.01$ (unpaired $t$-test and ANOVA). and mitochondrial dysfunction (27). The NAD ${ }^{+}$-dependent deacetylase SIRT1 can increase mammalian FOXO3 transcription factor through direct binding or deacetylation to induce cell resistance to oxidative stress (28), which implies that $A \beta$-induced inhibition of SIRT1 expression may contribute to the reduction of MTS in cells as a result of the excessive generation of ROS. Our results are consistent with a recent report (29) that suggested $A \beta$-mediated SIRT1 inhibition may contribute to decreased cell viability, but the mechanism by which $A \beta$ regulates SIRT1 expression is largely unknown.

This study demonstrated that $A \beta$-induced IL-8, IL-6, and MMP-9 expressions were attenuated in cells pretreated with SIRT1 activators, and the A $\beta$-induced proinflammatory effects were exacerbated by knockdown of SIRT1 expression (Figure 3). These results suggested that SIRT1 could negatively regulate $A \beta$-induced inflammation. A recent study also reported that the SIRT1 activator resveratrol prevents the proinflammatory effect of $A \beta$ on macrophages (30).

Exposure of RPE cells to $A \beta$ resulted in a disruption of ZO-1 and occludin (Figure 4), loss of TER (Figure 5A), and increased permeability (Figure 5B). Furthermore, the SIRT1 activator SRT1720 inhibited deleterious effects of $A \beta$ on morphology (Figure 4) and barrier function of RPE monolayers (Figure 5), and knockdown SIRT1 expression aggravated $A \beta$-induced RPE barrier disruption (Figures 4 and 5). A $\beta$-induced MMP-9 expression may contribute to barrier disruption $(11,31)$; therefore, transcriptional inhibition of MMP-9 by SIRT1, which is a negative regulator of MMP-9 (25), may protect RPE cells from A $\beta$-induced RPE barrier disruption via inhibition of MMP-9 expression. $A \beta$ induced a diffuse ezrin staining at both sides of RPE cells, but activation of SIRT1 did not attenuate the effect of
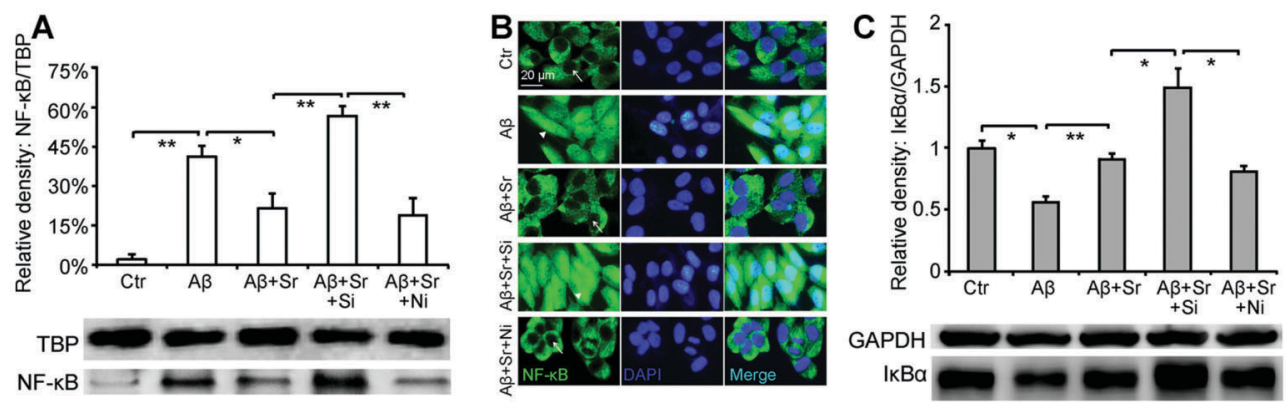

Figure 6. Detection of $\mathrm{NF}-\kappa \mathrm{B} / \mathrm{p} 65$ and I $\mathrm{\kappa Ba}$ expression. $A$, Western blot analysis of $\mathrm{NF}-\kappa \mathrm{B} / \mathrm{p} 65$ expression in the nucleus. $B$,

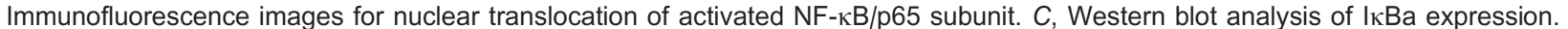
Exposure to $\mathrm{OA} \beta$ induced a significant increase of $\mathrm{NF}-\mathrm{kB} / \mathrm{p} 65$ protein level in the nucleus and markedly increased nuclear NF-kB/p65 staining. In addition, the protein level of IкBa was decreased in OA $\beta$-stimulated cells. SRT1720 significantly abrogated the effect of

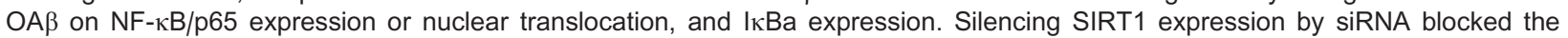
inhibitory effect of SRT1720 on OA $\beta$-induced NF-kB/p65 expression and activation, but it also significantly increased the protein level of $\mathrm{I} \mathrm{KB}$. Relative protein expression was quantified by calculating the mean gray density value. Green fluorescence indicates the location of the p65 subunit; nuclei are stained with DAPI (blue). Sr: cells treated with SRT1720; Si: cells transfected with SIRT1 siRNA; Ni: cells transfected with negative control siRNA; TBP: TATA binding protein; GAPDH: glyceraldehyde-3-phosphate dehydrogenase. *P $<0.05$, ${ }^{* *} \mathrm{P}<0.01$ (unpaired $t$-test and ANOVA). 
$A \beta$ on localization of ezrin (Figure 4). This result implied that $A \beta$ stimulation could destroy the well-developed polarization of normal RPE monolayers, but it was independent of SIRT1 inhibition. Although diffuse immune staining of ezrin has been reported in atrophic and hyperplastic RPE cells of patients (20), the essential factor that controls localization of ezrin or polarization of RPE is largely unknown.

Western blot analysis of NF- $\mathrm{NB}$ and $\mathrm{I} \kappa \mathrm{Ba}$ as well as immunofluorescence of $\mathrm{NF}-\kappa \mathrm{B} / \mathrm{p} 65$ suggested that SRT1720 protected RPE cells from the A $\beta$-mediated breakdown of barrier integrity via its activation of SIRT1 (Figure 6). SIRT1 then suppressed NF-kB activation $(16,17)$, which is required for transcriptional regulation of IL-6, IL-8, and MMP-9.

Therefore, we conclude that SIRT1 activation attenuated $A \beta$-induced inflammation by suppressing $N F-\kappa B$ activation, the transcription of which regulates expression of IL-6, IL-8, and MMP-9 (Figure 7A). The prototypical NF$\kappa \mathrm{B}$ complex, including a heterodimer of p50 and p65 subunits, is chiefly sequestered in the cytoplasm through its association with $1 \kappa B \alpha$ (32). The I kappa B kinase (IKK) complex, including two catalytic subunits, IKK $\alpha$ and IKK $\beta$, as well as a regulatory subunit IKK $\gamma(N F-K B$ essential modulator), lies at the confluence of the different NF-kB signaling cascades: Toll-like receptors, T-cell receptor, or ataxia telangiectasia mutated protein. Stimulus-induced phosphorylation of the IKK complex mediates $\mathrm{I} K \mathrm{~B} \alpha$ activation or phosphorylation, this response in turn triggers the rapid ubiquitination and subsequent degradation of $\mid \kappa B \alpha$, and the newly liberated $N F-\kappa B$ heterodimer then rapidly translocates into the nucleus (33). Then, p300/CBP-mediated RelA acetylation increases transcrip-

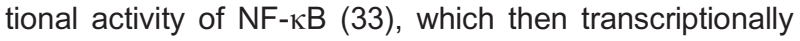
regulates genes encoding cytokines and MMPs. However, acetylated forms of RelA are deacetylated through specific interaction with SIRT1 (17). Deacetylation of RelA promotes its effective binding to $I \kappa B \alpha$ and leads to $I \kappa B \alpha-$ dependent nuclear export of NF-кB (33); therefore, SIRT1 could attenuate NF- $\mathrm{B}$ activation-mediated inflammation via deacetylation of RelA/p65. On the one hand, $A \beta$ could bind Toll-like receptor 4 (30) to activate the classical NF-кB signaling pathway; and, on the other hand, $A \beta$ could increase NF- $\kappa B$ activation via inhibition of SIRT1 (Figure $7 A)$. Then, NF- $\kappa B$ activation induced by $A \beta$ transcriptionally regulates IL-6, IL-8, and MMP-9 $(34,35)$. In general, A $\beta$, detected in drusen of AMD (3-5), may trigger inflammatory responses in the RPE/choroidal layers of the eye for the following reasons (Figure 7B): 1) The retina is an immuneprivileged site where inflammatory responses are suppressed, but opening of epithelial barriers by MMP-9 may be a mechanism that allows passage of plasma proteins and inflammatory cells into this privileged compartment. 2) The aqueous humor of AMD patients contains higher concentrations of IL-6 and IL-8 (15), which play pivotal roles in NK cell and neutrophil recruitment. It has been demonstrated that the two kinds of immune cells could accelerate tissue damage via release of ROS or directly
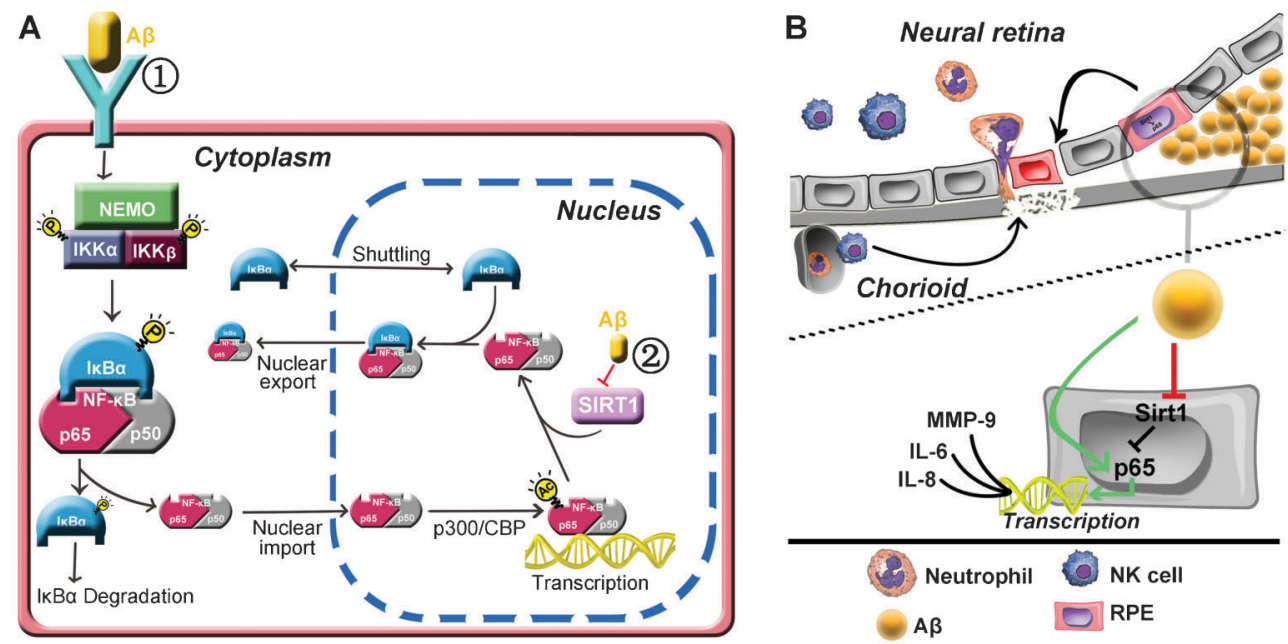

Figure 7. Schematic illustration of the effect of OA $\beta$ on the NF-kB/SIRT1 pathway in cells and the role of OA $\beta$ in the chronic inflammation of AMD in tissue. $A, A \beta$ induces NF-кB signaling via inhibition of SIRT1. 1: Binding of $A \beta$ to an extracellular receptor, such as TLR4, leads to phosphorylation of IKK complex subunits (NEMO, IKK $\alpha$ and IKK $\beta$ ), which subsequently phosphorylate IKB $\alpha$ and lead to its proteosomal degradation. This then releases NF-kB into the nucleus. 2: Newly liberated NF-kB can be acetylated for fulltranscriptional activation. However, the acetylated NF-kB can also be deacetylated by SIRT1. This action of SIRT1 promotes IкB $\alpha$

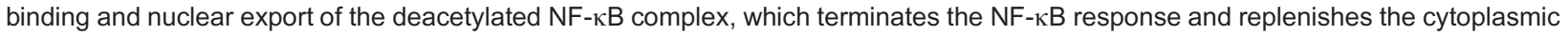
pool of latent NF- $\mathrm{KB} / \mathrm{I} \kappa \mathrm{B} \alpha$ complexes. $B$, A $\beta$-induced expression of IL-8, IL-6 and MMP-9 contributes to chronic inflammation in agerelated macular degeneration. Figure adapted from Refs. 11,33,38. 
inducing apoptosis of cells $(36,37)$. 3) Continued presence (sometimes over many years) of proinflammatory factors and immune cells in the retina may cause chronic inflammation. Together with these observations, our present results demonstrated that $A \beta$-induced inflammation and RPE barrier disruption were regulated by the SIRT1/NF-kB pathway. Maintenance of barrier integrity in the RPE by blocking the action of $A \beta$ or by activation of SIRT1 may thus represent a new approach to the treatment of AMD.

\section{References}

1. Buschini E, Piras A, Nuzzi R, Vercelli A. Age related macular degeneration and drusen: neuroinflammation in the retina. Prog Neurobiol 2011; 95: 14-25, doi: 10.1016/ j.pneurobio.2011.05.011.

2. Johnson LV, Forest DL, Banna CD, Radeke CM, Maloney $\mathrm{MA}, \mathrm{Hu} \mathrm{J}$, et al. Cell culture model that mimics drusen formation and triggers complement activation associated with age-related macular degeneration. Proc Natl Acad Sci U S A 2011; 108: 18277-18282, doi: 10.1073/pnas.1109703108.

3. Luibl V, Isas JM, Kayed R, Glabe CG, Langen R, Chen J. Drusen deposits associated with aging and age-related macular degeneration contain nonfibrillar amyloid oligomers. J Clin Invest 2006; 116: 378-385, doi: 10.1172/JCl25843.

4. Mullins RF, Russell SR, Anderson DH, Hageman GS. Drusen associated with aging and age-related macular degeneration contain proteins common to extracellular deposits associated with atherosclerosis, elastosis, amyloidosis, and dense deposit disease. FASEB J 2000; 14: 835-846.

5. Isas JM, Luibl V, Johnson LV, Kayed R, Wetzel R, Glabe CG, et al. Soluble and mature amyloid fibrils in drusen deposits. Invest Ophthalmol Vis Sci 2010; 51: 1304-1310, doi: 10.1167 /iovs.09-4207.

6. Jo $\mathrm{DH}, \mathrm{Kim} \mathrm{JH}, \mathrm{Kim} \mathrm{JH}$. How to overcome retinal neuropathy: the fight against angiogenesis-related blindness. Arch Pharm Res 2010; 33: 1557-1565, doi: 10.1007/ s12272-010-1007-6.

7. Cunha-Vaz J, Bernardes R, Lobo C. Blood-retinal barrier. Eur J Ophthalmol 2011; 21 (Suppl 6): S3-S9, doi: 10.5301/ EJO.2010.6049.

8. Bailey TA, Kanuga N, Romero IA, Greenwood J, Luthert PJ, Cheetham ME. Oxidative stress affects the junctional integrity of retinal pigment epithelial cells. Invest Ophthalmol Vis Sci 2004; 45: 675-684, doi: 10.1167/ iovs.03-0351.

9. Peng S, Gan G, Rao VS, Adelman RA, Rizzolo LJ. Effects of proinflammatory cytokines on the claudin-19 rich tight junctions of human retinal pigment epithelium. Invest Ophthalmol Vis Sci 2012; 53: 5016-5028, doi: 10.1167/ iovs.11-8311.

10. Bruban J, Glotin AL, Dinet V, Chalour N, Sennlaub F, Jonet $\mathrm{L}$, et al. Amyloid-beta(1-42) alters structure and function of retinal pigmented epithelial cells. Aging Cell 2009; 8: 162177, doi: 10.1111/j.1474-9726.2009.00456.x.

11. Cao L, Wang $\mathrm{H}$, Wang $\mathrm{F}$. Amyloid-beta-induced matrix metalloproteinase- 9 secretion is associated with retinal pigment epithelial barrier disruption. Int J Mol Med 2013; 31: 1105-1112.

12. Johnson LV, Leitner WP, Rivest AJ, Staples MK, Radeke

\section{Acknowledgments}

We thank the Biochemistry and Molecular Biology Institute of Shanghai Tenth People's Hospital and are grateful for their technological support. Research supported by the Science and Technology Commission of Shanghai (\#11JC1409900) and the National High Technology Research and Development Program of China (863 Program, \#S2010GR0002).

MJ, Anderson DH. The Alzheimer's A beta-peptide is deposited at sites of complement activation in pathologic deposits associated with aging and age-related macular degeneration. Proc Natl Acad Sci U S A 2002; 99: 1183011835, doi: 10.1073/pnas.192203399.

13. Wang J, Ohno-Matsui K, Yoshida T, Kojima A, Shimada N, Nakahama K, et al. Altered function of factor I caused by amyloid beta: implication for pathogenesis of age-related macular degeneration from Drusen. J Immunol 2008; 181: 712-720.

14. Kurji KH, Cui JZ, Lin T, Harriman D, Prasad SS, Kojic L, et al. Microarray analysis identifies changes in inflammatory gene expression in response to amyloid-beta stimulation of cultured human retinal pigment epithelial cells. Invest Ophthalmol Vis Sci 2010; 51: 1151-1163, doi: 10.1167/iovs.09-3622.

15. Jonas JB, Tao Y, Neumaier M, Findeisen P. Cytokine concentration in aqueous humour of eyes with exudative age-related macular degeneration. Acta Ophthalmol 2012; 90: e381-e388, doi: 10.1111/j.1755-3768.2012.02414.x.

16. Chen L, Fischle W, Verdin E, Greene WC. Duration of nuclear NF-kappaB action regulated by reversible acetylation. Science 2001; 293: 1653-1657, doi: 10.1126/science.1062374.

17. Yeung F, Hoberg JE, Ramsey CS, Keller MD, Jones DR, Frye RA, et al. Modulation of NF-kappaB-dependent transcription and cell survival by the SIRT1 deacetylase. EMBO J 2004; 23: 2369-2380, doi: 10.1038/sj.emboj.7600244.

18. Howitz KT, Bitterman KJ, Cohen HY, Lamming DW, Lavu S, Wood JG, et al. Small molecule activators of sirtuins extend Saccharomyces cerevisiae lifespan. Nature 2003; 425: 191196, doi: 10.1038/nature01960.

19. Kayed R, Head E, Thompson JL, McIntire TM, Milton SC, Cotman $\mathrm{CW}$, et al. Common structure of soluble amyloid oligomers implies common mechanism of pathogenesis. Science 2003; 300: 486-489, doi: 10.1126/ science. 1079469.

20. Kivela T, Jaaskelainen J, Vaheri A, Carpen O. Ezrin, a membrane-organizing protein, as a polarization marker of the retinal pigment epithelium in vertebrates. Cell Tissue Res 2000; 301: 217-223, doi: 10.1007/s004410000225.

21. Villalba JM, Alcain FJ. Sirtuin activators and inhibitors. Biofactors 2012; 38: 349-359, doi: 10.1002/biof.1032.

22. Feng $\mathrm{S}$, Cen J, Huang $\mathrm{Y}$, Shen $\mathrm{H}$, Yao L, Wang $\mathrm{Y}$, et al. Matrix metalloproteinase-2 and -9 secreted by leukemic cells increase the permeability of blood-brain barrier by disrupting tight junction proteins. PLOS One 2011; 6: e20599, doi: 10.1371/journal.pone.0020599.

23. Martins T, Baptista S, Goncalves J, Leal E, Milhazes N, Borges $F$, et al. Methamphetamine transiently increases the 
blood-brain barrier permeability in the hippocampus: role of tight junction proteins and matrix metalloproteinase-9. Brain Res 2011; 1411: 28-40.

24. Bauer AT, Burgers HF, Rabie T, Marti HH. Matrix metalloproteinase-9 mediates hypoxia-induced vascular leakage in the brain via tight junction rearrangement. $J$ Cereb Blood Flow Metab 2010; 30: 837-848, doi: 10.1038/ jcbfm.2009.248.

25. Nakamaru $\mathrm{Y}$, Vuppusetty $\mathrm{C}$, Wada $\mathrm{H}$, Milne JC, Ito $\mathrm{M}$, Rossios C, et al. A protein deacetylase SIRT1 is a negative regulator of metalloproteinase-9. FASEB J 2009; 23: 28102819, doi: 10.1096/fj.08-125468.

26. Li F, Gong Q, Dong H, Shi J. Resveratrol, a neuroprotective supplement for Alzheimer's disease. Curr Pharm Des 2012; 18: 27-33, doi: 10.2174/138161212798919075.

27. Canevari L, Abramov AY, Duchen MR. Toxicity of amyloid beta peptide: tales of calcium, mitochondria, and oxidative stress. Neurochem Res 2004; 29: 637-650, doi: 10.1023/ B:NERE.0000014834.06405.af.

28. Brunet A, Sweeney LB, Sturgill JF, Chua KF, Greer PL, Lin $\mathrm{Y}$, et al. Stress-dependent regulation of FOXO transcription factors by the SIRT1 deacetylase. Science 2004; 303: 20112015, doi: 10.1126/science.1094637.

29. Feng $X$, Liang $N$, Zhu D, Gao Q, Peng L, Dong $H$, et al. Resveratrol inhibits beta-amyloid-induced neuronal apoptosis through regulation of SIRT1-ROCK1 signaling pathway. PLoS One 2013; 8: e59888, doi: 10.1371/journal.pone.0059888.

30. Capiralla H, Vingtdeux V, Zhao H, Sankowski R, Al-Abed $Y$, Davies $\mathrm{P}$, et al. Resveratrol mitigates lipopolysaccharide- and Abeta-mediated microglial inflammation by inhibiting the TLR4/NF-kappaB/STAT signaling cascade. J Neurochem
2012; 120: 461-472, doi: 10.1111/j.1471-4159.2011.07594.x.

31. Kook SY, Hong HS, Moon M, Ha CM, Chang S, Mook-Jung I. Abeta(1)(-)(4)(2)-RAGE interaction disrupts tight junctions of the blood-brain barrier via $\mathrm{Ca}(2)(+)$-calcineurin signaling. $J$ Neurosci 2012; 32: 8845-8854, doi: 10.1523/ JNEUROSCI.6102-11.2012.

32. Baldwin AS Jr. The NF-kappa B and I kappa B proteins: new discoveries and insights. Annu Rev Immunol 1996; 14: 649683, doi: 10.1146/annurev.immunol.14.1.649.

33. Chen LF, Greene WC. Regulation of distinct biological activities of the NF-kappaB transcription factor complex by acetylation. J Mol Med 2003; 81: 549-557, doi: 10.1007/ s00109-003-0469-0.

34. Park SY, Jin ML, Kim YH, Kim Y, Lee SJ. Anti-inflammatory effects of aromatic-turmerone through blocking of NFkappaB, JNK, and p38 MAPK signaling pathways in amyloid beta-stimulated microglia. Int Immunopharmacol 2012; 14 : 13-20, doi: 10.1016/j.intimp.2012.06.003.

35. Walker DG, Lue LF, Beach TG. Gene expression profiling of amyloid beta peptide-stimulated human post-mortem brain microglia. Neurobiol Aging 2001; 22: 957-966, doi: 10.1016/ S0197-4580(01)00306-2.

36. Nathan CF. Neutrophil activation on biological surfaces. Massive secretion of hydrogen peroxide in response to products of macrophages and lymphocytes. J Clin Invest 1987; 80: 1550-1560, doi: 10.1172/JCl113241.

37. Lowin B, Peitsch MC, Tschopp J. Perforin and granzymes: crucial effector molecules in cytolytic T lymphocyte and natural killer cell-mediated cytotoxicity. Curr Top Microbiol Immunol 1995; 198: 1-24, doi: 10.1007/978-3-642-79414-8_1.

38. Tilstra JS, Clauson CL, Niedernhofer LJ, Robbins PD. NFkappaB in aging and disease. Aging Dis 2011; 2: 449-465. 